\title{
Mechanisms of Hepatitis B Virus Resistance Development
}

\author{
Nadia Warner Stephen Locarnini \\ Research and Molecular Development, Victorian Infectious Diseases Reference Laboratory (VIDRL), \\ North Melbourne, Vic., Australia
}

\section{Key Words}

Hepatitis B virus - Antiviral resistance $\cdot$ Mechanisms of action · Nucleos $(\mathrm{t})$ ide analogues

\begin{abstract}
Hepatitis B virus (HBV) resistance to nucleos(t)ide analogue (NA) therapy is essentially structure specific, with each NA falling within three main structural groups. Resistance to each of these is characterized by specific mutations in the reverse transcriptase domains of the HBV polymerase, and may be associated with compensatory mutations which can increase replication. HBV polymerase is considered to have a traditional 'right-handed' structural conformation, and each of the resistance mutations is predicted to cause a specific structural change of the polymerase, thereby preventing incorporation of NA into replicating DNA. The selection of resistance occurs at different rates for each NA, and is affected by the high mutational rate of $\mathrm{HBV}$ and the ability of the drug to suppress viral replication. Some mutations or combinations of mutations may be associated with multidrug resistance, limiting treatment options. In contrast to most other viruses, resistance in HBV is confounded by the overlapping surface gene, the major NA-resistant mutations also altering the surface proteins in most cases, potentially altering virus secretion and neutralization, which may pose a public health threat in the future.

(c) 2014 S. Karger AG, Basel
\end{abstract}

() 2014 S. Karger AG, Basel

0300-5526/14/0574-0218\$39.50/0

\section{Introduction to Nucleos(t)ide Analogue Resistance}

Resistance and cross-resistance to nucleos(t)ide analogues (NA) is essentially structure specific, and the five approved NAs can be considered under three main structural groups (table 1): (1) L-nucleoside analogues, including lamivudine (LMV), its 5-fluoro derivative emtricitabine and telbivudine (L-dT), (2) acyclic nucleoside phosphonates, represented by the dAMP analogues adefovir (ADV) and tenofovir (TFV), and (3) deoxyguanosine analogues in which the deoxyribose moiety is replaced by a cyclopentane sugar derivative, including entecavir (ETV).

\section{Resistance to LMV and Other L-Nucleosides}

LMV resistance occurs at a cumulative rate of about $14-20 \%$ per year achieving levels of over $70 \%$ by $5-6$ years of monotherapy $[1,2]$. This rate is even higher in individuals who are coinfected with HIV-1 [3]. Mutations that result in replacement of methionine in the tyrosinemethionine-aspartate (YMDD) catalytic site motif with either valine or isoleucine confer resistance to $\operatorname{LMV}[4,5]$. These changes are designated rtM204V/I, using the genotype-independent nomenclature system proposed previously [6]. rtM204I substitution has been detected in isolation, but the rtM204V change in the HBV DNA in those patients failing LMV therapy is found in association with

\section{KARGER}

E-Mail karger@karger.com

www.karger.com/int
Stephen Locarnini

Victorian Infectious Diseases Reference Laboratory

10 Wreckyn Street

North Melbourne, VIC 3051 (Australia)

E-Mail stephen.locarnini@mh.org.au 
Table 1. Patterns and pathways of antiviral drug resistance in chronic hepatitis B in the context of cross-resistance

\begin{tabular}{lllllll}
\hline Pathway & $\begin{array}{l}\text { Amino acid substitutions } \\
\text { in the rt domain }\end{array}$ & LMV & LdT & ETV & ADV & TFV \\
& Wild-type & $\mathrm{S}$ & $\mathrm{S}$ & $\mathrm{S}$ & $\mathrm{S}$ & $\mathrm{S}$ \\
L-nucleoside (LMV/L-dT) & M204I/V & $\mathrm{R}$ & $\mathrm{R}$ & $\mathrm{I}$ & $\mathrm{S}$ & $\mathrm{S}$ \\
Acyclic phosphonate (ADV) & $\mathrm{N} 236 \mathrm{~T}$ & $\mathrm{~S}$ & $\mathrm{~S}$ & $\mathrm{~S}$ & $\mathrm{R}$ & $\mathrm{I}$ \\
Shared (LMV, L-dT, ADV) & A181T/V & $\mathrm{R}$ & $\mathrm{R}$ & $\mathrm{S}$ & $\mathrm{R}$ & $\mathrm{I}$ \\
Double (ADV, TFV) & A181T/V+N236T & $\mathrm{R}$ & $\mathrm{R}$ & $\mathrm{S}$ & $\mathrm{R}$ & $\mathrm{R}$ \\
D-cyclopentane (ETV) & L180M+M204V/I & $\mathrm{R}$ & $\mathrm{R}$ & $\mathrm{R}$ & $\mathrm{S}$ & $\mathrm{S}$ \\
& $\pm \mathrm{I} 169 \pm \mathrm{T} 184$ & & & & & \\
\hline
\end{tabular}

$\mathrm{I}=$ Intermediate sensitivity; $\mathrm{R}=$ resistant; $\mathrm{S}=$ sensitive. $\mathrm{I}, \mathrm{R}$, and $\mathrm{S}$ are based on cell culture and clinical responses. Modified from [29, 30, 50].

other changes, which are essentially compensatory; these include rtL180M and rtV173L [7-9], and rtL80V/I [10]. These compensatory changes typically result in enhanced viral replication in the setting of primary resistance. Phenotyping of these compensatory changes has shown that they only enhance replication and have no direct effect on antiviral drug resistance [10-15].

The second group of substitutions affecting L-nucleoside antiviral sensitivity have been found in the B domain and include the rtA181T substitution [16]. This change is also associated with ADV resistance [17], thereby making it a multidrug-resistant marker. L-dT ( $\beta$-L- $2^{\prime}$-deoxythymidine) has a similar resistance profile to LMV, with the rtM204I substitution being the major codon change associated with drug failure [18]. Other changes such as rtA181T have also been detected.

\section{Resistance to Acyclic Nucleoside Phosphonates}

ADV Resistance

ADV resistance emerges more slowly than LMV, occurring at a cumulative annual rate of approximately $2.5 \%$ over 4 years $[19,20]$, although long-term (5-year) studies indicate higher rates in HBeAg-negative patients with chronic hepatitis B [21]; it is also more frequent in individuals when used as rescue therapy after LMV failure [22]. Single nucleotide changes that produce rtN236T and/or rtA181V/T substitutions are sufficient to cause clinical failure of $\operatorname{ADV}[17,21,23,24]$.

\section{TFV Resistance}

TFV resistance has been linked to the rtA194T substitution, but only in association with changes that cause LMV resistance and typically in the setting of coinfection with HIV-1 [25]. Results of recent clinical studies suggest that TFV is less effective in the setting of ADV resistance $[26,27]$, but no specific single substitution has been identified that can be unequivocally linked to primary TFV resistance.

\section{Resistance to ETV}

Resistance to ETV has been mainly reported in patients who have had previous LMV failure. Sequencing $\mathrm{HBV}$ isolates from these patients usually reveals mutations that cause additional amino acid substitutions in association with the classical rtL180M/rtM204V LMV resistance changes [28]. These changes include at least one of rtI169T, rtT184G, rtS202I, or rtM250V. The overall resistance rate for ETV in naïve patients is $<1 \%$ at $5-6$ years $[29,30]$.

\section{Resistance to More than One NA}

Of the 8 codons associated with primary drug resistance (rtI169T, rtT184G, rtL180M, rtA181T/V, rtS202I, rtM204V/I, rtN236LT, and rtM250V), only the rtA181T and rtL180M+rtM204V substitutions have been definitely associated with multidrug resistance.

\section{Molecular Mechanisms of Resistance to NAs}

\section{HBV Polymerase}

$\mathrm{HBV}$ polymerase is a multifunctional protein that has four domains: a priming region, a spacer region of unknown function, a catalytic region that functions as a RNA-dependent RNA polymerase/DNA polymerase, and a carboxy terminal region that has ribonuclease $\mathrm{H}$ 
activity [31]. Although a crystal structure of HBV polymerase has not been published, much of its structure has been deduced from homology with HIV-1 reverse transcriptase (RT) [32-34]. It has been possible to create 3 -dimensional models of the reverse transcriptase (rt) region of HBV polymerase based on this homology. Thus, the amino acid changes resulting from mutations that confer antiviral resistance can be mapped to functional regions to provide a better understanding of mechanisms for resistance $[35,36]$. Most polymerases can be considered to have a common right-handed configuration comprising a thumb, palm, and finger domain (fig. 1a) $[37,38]$. The palm domain includes the active site and catalyzes the phosphoryl transfer reaction, while the finger domain facilitates interactions with the incoming dNTPs as well as the template base to which it is paired. The thumb domain plays a role in positioning the duplex DNA facilitating increased processivity and translocation, as well as acting as a primer domain [38]. NAs and dNTPs bind at a site that is located in the palm subdomain adjacent to the $3^{\prime}$ terminus of the primer strand [39]. Unlike HIV, the HBV rt shows a preference for nucleotides with the L-configuration in contrast to other polymerases that prefer nucleotides with the $\mathrm{D}$-configuration [40].

A molecular mechanism of resistance for LMV has been proposed based on the crystal structure of the HIV RT $[32,33]$. LMV binds at a pocket in the surface of the HBV rt (palm region) formed in part by residue rtM204 [5]. The primary LMV substitutions rtM204V/I are predicted to reduce the accessible surface area between the HBV rt and LMV because the methyl group on the $\beta$-branched side chain of valine/isoleucine now impinges on the sulfur atom in the unnatural L-oxathiolane ring of LMV (fig. 1b) [5, 36, 41]. These structural alterations have two main effects: (1) steric hindrance to decrease binding of LMV to the viral polymerase and (2) reduced catalytic activity to incorporate LMV-triphosphate into replicating viral DNA $[5,36,41]$. These substitutions do not affect the binding of the natural substrate, cytosine, to the viral polymerase, and, therefore, the polymerase is still active in viral replication.

A molecular mechanism of resistance to ADV has also been proposed based on these homology models focusing on the $\mathrm{A}$ and $\mathrm{D}$ domains for how the most common $\mathrm{ADV}$ mutation, rtN236T, which is outside the catalytic site, leads to ADV resistance (fig. 1c) [23]. Domains A and D are thought to be involved in dNTP binding and the modelling has suggested that rtN236 in the D domain may be hydrogen bonded to the physically adjacent rtS85 [42] from the A domain, interacting directly with the $\gamma$ phosphate of ADV diphosphate. The rtN236T mutation may disrupt the hydrogen bond between rtN236 and rtS85 and also between rtN236 and ADV diphosphate, thereby decreasing the binding affinity for ADV [42]. In contrast, mutations that affect the $\alpha$-helix of the B domain appear to alter the positioning of the nucleic acid template strand relative to the catalytic region. The rtA181T/V changes may bend the helices through hydrogen bond formation, and drug resistance could easily result from an allosteric change in the catalytic site $[35,43]$.

For TFV, residue rtA194 is located in a loop at the end of the B domain, which contains the $\alpha$-helix that interacts with the nucleic acid template. The rtA194T substitution may affect polymerization efficiency by causing allosteric changes that result in misalignment between the template and dNTP-binding site. Molecular modeling of the rt194 codon has revealed that the residue is located in the hinge of an $\alpha$-helix that forms the B domain $\alpha$-helix (the DNA template-binding region) with the $\beta$-sheet of the $C$ domain [25]. rtA194T may alter the position of the DNA template strand relative to the dNTP-binding site and thereby affect DNA polymerization [25].

For ETV resistance, molecular modelling has suggested two possible pathways with quite different mechanisms for ETV resistance. Modelling of the first group of changes at positions rt 169 and rt 250 reveals that these codon alternations have an impact on the primer-binding region of the RT, which interacts with the $3^{\prime}$ terminus of the DNA template. The rt250 mutation appears to alter the binding interaction between the primer strand and the template DNA. rt169 interacts with dNTP and may work cooperatively with the mutation at rt250. The net effect of these mutations may alter elongation of the DNA strand. In vitro studies have extended these predictions; for example, the rtM250V in the absence of other changes confers low-level resistance to ETV, but leads to a $>250$-fold increase in resistance to ETV in the presence of the LMV rtM204V mutation [28]. Addition of the third mutation, rtI169T, further increases resistance to ETV $>750$-fold, but has no effect by itself [28]. Interestingly, the rtM250V mutation in combination with the rtM204V mutation significantly impairs HBV replication, but addition of the rtI169T mutation appears to improve HBV replication and functions as an adaptive compensatory change as well [28]. Modelling of the second group of substitutions reveals an altered geometry of the polymerase nucleotide-binding pocket near the YMDD site. The rtT184G and rtS202I mutations could interact and thereby affect the hydrophobic 


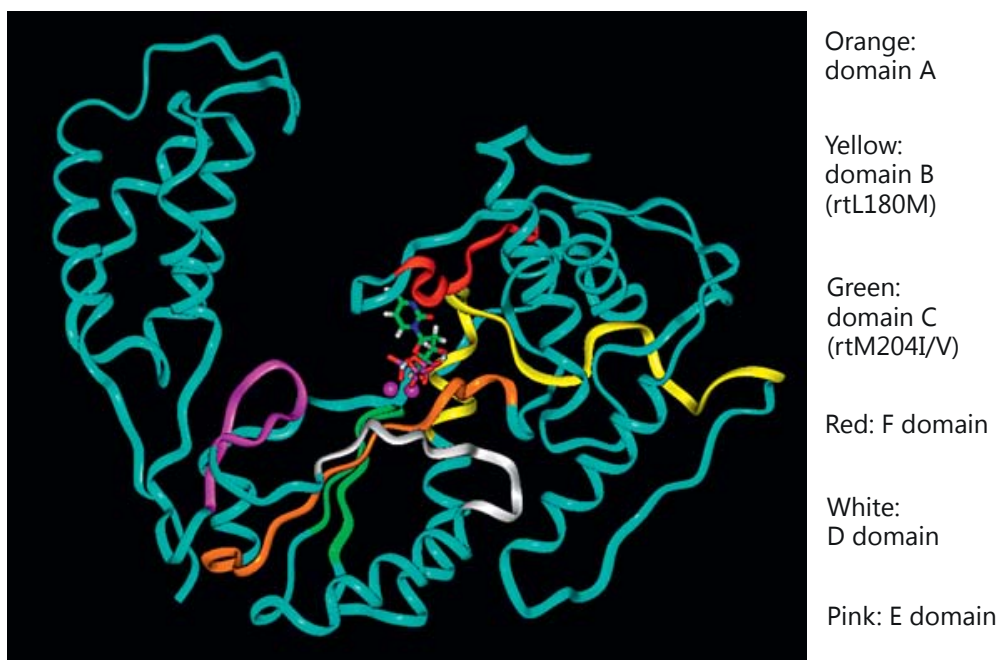

Orange:

Yellow:

domain B

(rtL180M)

Green:

domain $\mathrm{C}$

Red: F domain

White:

Pink: E domain

Wt type polymerase + LMV

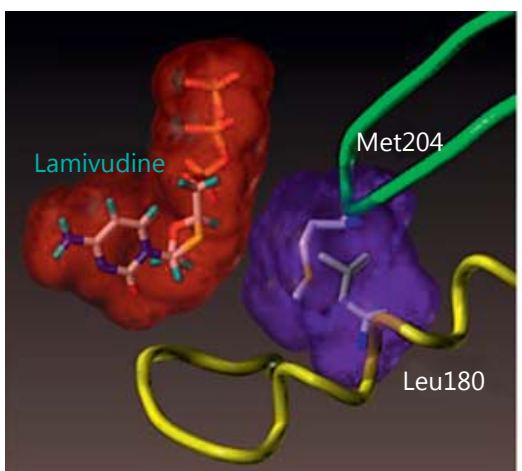

Wild-type rtN236

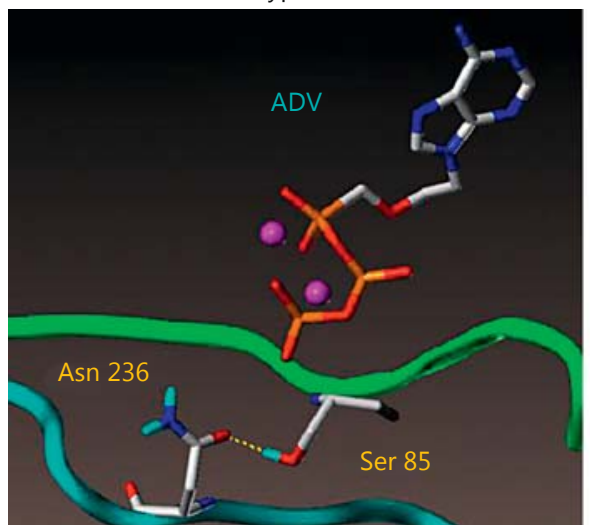

Steric hindrance with HBV polymerase mutants + LMV

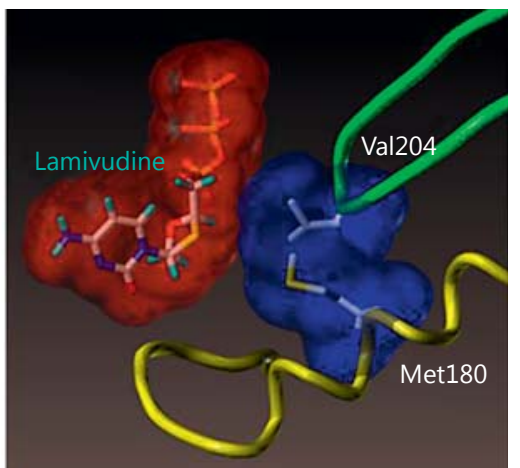

rtN236T

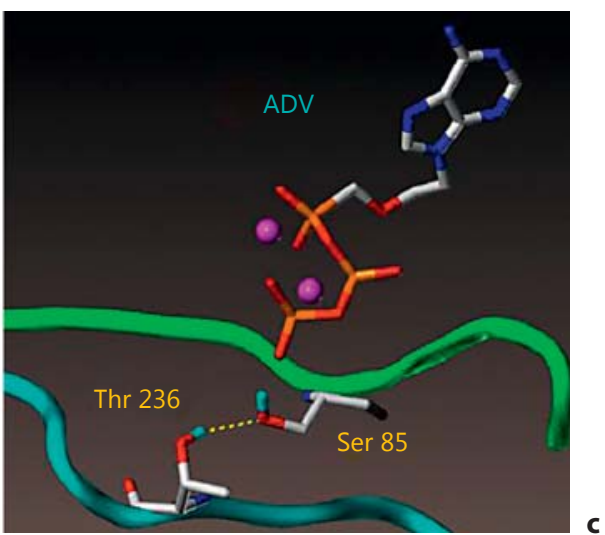

Fig. 1. a Homology model of HBV polymerase based on HIV RT. b Molecular modelling showing steric hindrance with HBV rtM204V and LMV (right). c Molecular modelling showing altered geometry caused by rtN236T, resulting in A-D domain perturbation. Modified from [35].

core region between the a-helix of the DNA templatebinding region and the $\beta$-sheet, which encodes the two catalytic aspartate residues in the $\mathrm{C}$ domain. The substitutions at residues rt 184 and rt202 are predicted to magnify the impact of the LMV-resistant M204V in the
YMDD loop, affecting substrate discrimination upon nucleotide loading and addition to the primer terminus. These changes would have to interact cooperatively with the rtM204V to provide a structural basis for the observed cross-resistance between the two drugs. Recom- 


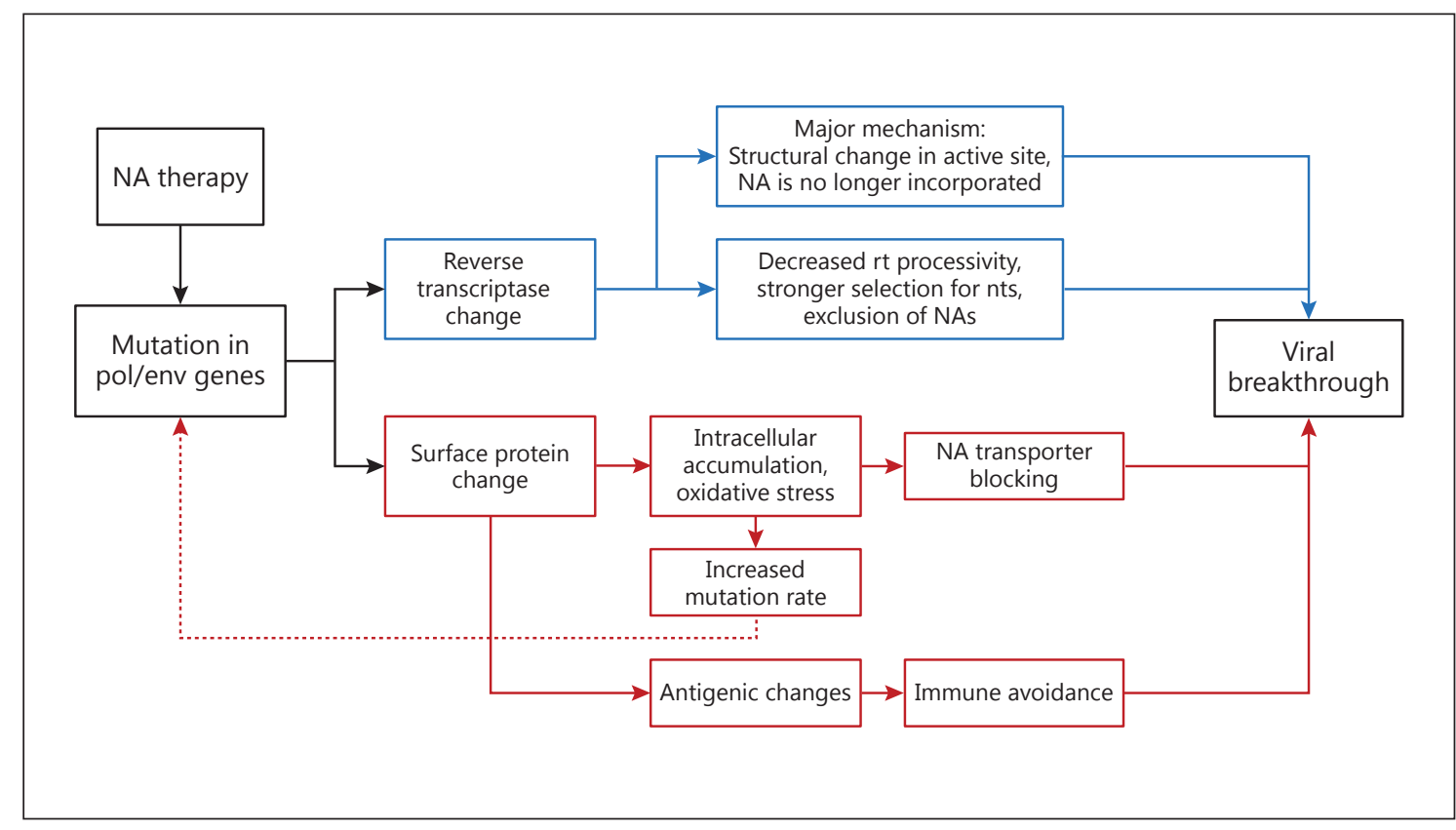

Fig. 2. Flow chart depicting the major pathway to resistance, and other mechanisms that may contribute to viral rebound.

binant constructs containing the LMV-resistant mutations $\mathrm{rtL} 180 \mathrm{M}$ and $\mathrm{rtM} 204 \mathrm{~V}$ in addition to either rtT184G or rtS202I demonstrated only a modest increase in resistance to ETV, approximately 40-fold [28]. However, when all four substitutions are present, resistance to ETV is increased $>750$-fold [28]. In the absence of the LMV-resistant mutations, the rtT184G and rtS202I mutations do not affect susceptibility of HBV to ETV in vitro [28].

\section{Virological Basis for Resistance}

HBV has a high rate of replication, with $10^{12}$ virions produced per day and a high mutational rate of approximately $10^{-5}$ substitutions/base/cycle [44]. This translates to approximately $10^{10-11}$ point mutations produced per day in individuals with active replication. Because the HBV genome is only approximately 3,200 base pairs, all possible single base changes can be produced per day. The HBV rt does not have a proofreading function to repair incorrectly incorporated nucleotides. Therefore, point mutations can arise very rapidly as do G-A hypermutations. Prior to therapy, there is a diverse swarm of viruses (quasi-species), including mutants with single and dou- ble mutations potentially associated with drug resistance. The probability of a mutation being selected during therapy depends on the ability of a drug to suppress viral replication. Hence, a drug with low antiviral activity does not exert substantial selection pressure on the virus, and the chance of drug resistance is not high. Conversely, complete suppression of viral replication allows little opportunity for resistance to emerge because mutagenesis is dependent on viral genome replication. NAs inhibit viral replication, but do not eliminate existing virus or affect the intrahepatic cccDNA reservoir in any major way. Monotherapy exerting modest antiviral activity and directed at one single target site would result in the highest probability of selecting antiviral drug resistance. The ideal treatment regimen should have antiviral activities targeted at different sites to reduce the risk of selecting out drug-resistant species. Resistance emerges when replication occurs in the presence of the drug selection pressure. Therefore, if we could achieve complete suppression of replication, resistance would not be an issue. Other factors contributing to the emergence of drug resistance are genetic barriers to the development of mutations, mechanism of drug resistance, viral replication space, and various host factors involved in controlling viral replication $[29,30]$. 


\section{Pol-Env Overlap}

The polymerase gene overlaps the envelope gene and changes in the polymerase gene, which confer antiviral resistance can cause concomitant changes to the overlapping reading frame of the envelope gene. Thus, the major resistance mutations associated with LMV, ADV, ETV, and L-dT failure also have the potential of altering the C-terminal region of HBsAg. For example, changes associated with LMV and ETV resistance, such as the rtM204V, result in a change at sI195M in the HBsAg, while the rtM204I change that is associated with both LMV and L-dT, is linked to three possible HBsAg changes at sW196S and sW196L, or a termination codon sW196*. The effect of the main LMV resistance mutations on the altered antigenicity of $\mathrm{HBsAg}$ have been examined in vitro [45] and been shown to affect virus secretion and neutralization in animal models [46]. In binding assays, HBsAg expressing these NA-resistant associated residues have reduced anti-HBs binding [45] to a level which was similar to the classical vaccine escape mutant, sG145R, and thus have a high public health potential [47].

The rtA181T mutation selected by ADV and LMV results in a stop codon mutation at sW172*. The ADVresistant mutation at rtA181V results in a change at sL173F. HBV with mutations that result in a stop codon in the envelope gene such as those for LMV and ADV need to be rescued in trans with a low percentage of wild-type HBV in order to enable viral assembly and release [48]. The ADV resistance substitution rtN236T does not affect the envelope gene and overlaps with the stop codon at the end of the region encoding the surface antigen.
The ETV resistance-associated changes at rtI169T, rtS184G, and rtS202I also affect HBsAg and result in changes at sF161L, sL/V176G, and sV194F. The rtM250V is located after the end of HBsAg. The $\mathrm{sF} 161 \mathrm{~L}$ is located within the region that was defined as the 'a' determinant or major hydrophilic region, which includes amino acids 90-170 of the HBsAg [49]. This region forms highly conformational epitopes, characterized by multiple disulfide bonds formed from sets of cysteine residues at aa107-138, 137-149, and 139-147 [49], as well as up to seven prolines populated over this region. Since distal substitutions such as sE164D significantly affect anti-HBs binding [45], the influence of other changes to HBsAg that are driven by NA resistance, such as sF161L, needs investigation in order to determine the effect on the envelope structure and subsequent anti-HBs binding. Overall, the public health impact of these changes to the HBV envelope does appear quite significant. The various possible effects from the pathways of NA resistance are shown in figure 2.

\section{Summary and Future Directions}

Resistance will remain an important issue in the management of patients with chronic hepatitis B because long-term therapy with NAs appears to be required in the majority of patients. It is presently unknown whether combination therapy with the current approved first-line therapies (ETV or TFV) is needed because after 7-8 years of monotherapy with these agents, minimal or no resistance has been reported. However, as these patients are neither 'cured' nor 'controlled', emergence of resistance is always a veiled threat in chronic hepatitis B. Thus, new therapies against new targets are still needed to truly 'cure' chronic hepatitis B.

\section{References}

1 Lok AS, et al: Long-term safety of lamivudine treatment in patients with chronic hepatitis $\mathrm{B}$. Gastroenterology 2003;125:1714-1722.

2 Fung J, et al: Nucleoside/nucleotide analogues in the treatment of chronic hepatitis B. J Antimicrob Chemother 2011;66:2715-2725.

3 Benhamou, et al: Long-term incidence of hepatitis $\mathrm{B}$ virus resistance to lamivudine in human immunodeficiency virus-infected patients. Hepatology 1999;30:1302-1306.

4 Bartholomew MM, et al: Hepatitis-B-virus resistance to lamivudine given for recurrent infection after orthotopic liver transplantation. Lancet 1997;349:20-22.
5 Allen MI, et al: Identification and characterization of mutations in hepatitis B virus resistant to lamivudine. Lamivudine Clinical Investigation Group. Hepatology 1998;27: 1670-1677.

6 Stuyver LJ, et al: Nomenclature for antiviralresistant human hepatitis $B$ virus mutations in the polymerase region. Hepatology 2001; 33:751-757.

7 Delaney WE 4th, et al: The hepatitis B virus polymerase mutation $\mathrm{rtV} 173 \mathrm{~L}$ is selected during lamivudine therapy and enhances viral replication in vitro. J Virol 2003;77:1183311841.
8 Nakanishi H, et al: Polymerase domain B mutation is associated with hepatitis relapse during long-term lamivudine therapy for chronic hepatitis B. Intervirology 2005;48:381-388.

-9 Pai SB, et al: Emergence of a novel mutation in the FLLA region of hepatitis $B$ virus during lamivudine therapy. Antimicrob Agents Chemother 2005;49:2618-2624.

10 Ogata N, et al: Novel patterns of amino acid mutations in the hepatitis B virus polymerase in association with resistance to lamivudine therapy in Japanese patients with chronic hepatitis B. J Med Virol 1999;59:270-276. 
11 Chong Y, et al: Mechanism of antiviral activities of $3^{\prime}$-substituted L-nucleosides against 3TC-resistant HBV polymerase: a molecular modelling approach. Antivir Chem Chemother 2003;14:309-319.

$12 \mathrm{Fu}$ L, Cheng YC: Role of additional mutations outside the YMDD motif of hepatitis B virus polymerase in L(-)SddC (3TC) resistance. Biochem Pharmacol 1998;55:1567-1572.

13 Melegari M, Scaglioni PP, Wands JR: Hepatitis B virus mutants associated with $3 \mathrm{TC}$ and famciclovir administration are replication defective. Hepatology 1998;27:628-633.

14 Ono SK, et al: The polymerase L528M mutation cooperates with nucleotide binding-site mutations, increasing hepatitis B virus replication and drug resistance. J Clin Invest 2001; 107:449-455.

15 Warner N, et al: The L80I substitution in the reverse transcriptase domain of the hepatitis $B$ virus polymerase is associated with lamivudine resistance and enhanced viral replication in vitro. Antimicrob Agents Chemother 2007; 51:2285-2292.

16 Yeh CT, et al: Clearance of the original hepatitis $B$ virus YMDD-motif mutants with emergence of distinct lamivudine-resistant mutants during prolonged lamivudine therapy. Hepatology 2000;31:1318-1326.

17 Fung SK, et al: Adefovir-resistant hepatitis B can be associated with viral rebound and hepatic decompensation. J Hepatol 2005;43: 937-943.

18 Lai CL, et al: A 1-year trial of telbivudine, lamivudine, and the combination in patients with hepatitis $\mathrm{B}$ e antigen-positive chronic hepatitis B. Gastroenterology 2005; 129:528536.

19 Westland C, et al: Hepatitis B virus genotypes and virologic response in 694 patients in phase III studies of adefovir dipivoxil1. Gastroenterology 2003;125:107-16.

-20 Yuen MF, Lai CL: Adefovir dipivoxil in chronic hepatitis B infection. Expert Opin Pharmacother 2004;5:2361-2367.

21 Hadziyannis SJ, et al: Long-term therapy with adefovir dipivoxil for HBeAg-negative chronic hepatitis B. N Engl J Med 2005;352:26732681.

22 Lee YS, et al: Increased risk of adefovir resistance in patients with lamivudine-resistant chronic hepatitis B after 48 weeks of adefovir dipivoxil monotherapy. Hepatology 2006;43: 1385-13891.

23 Angus $P$, et al: Resistance to adefovir dipivoxil therapy associated with the selection of a novel mutation in the HBV polymerase. Gastroenterology 2003;125:292-297.
24 Villeneuve JP, et al: Selection of a hepatitis B virus strain resistant to adefovir in a liver transplantation patient. J Hepatol 2003;39: 1085-1089.

25 Sheldon J, et al: Selection of hepatitis B virus polymerase mutations in HIV-coinfected patients treated with tenofovir. Antivir Ther 2005; 10:727-734.

26 Patterson SJ, et al: Tenofovir disoproxil fumarate rescue therapy following failure of both lamivudine and adefovir dipivoxil in chronic hepatitis B. Gut 2011;60:247-254.

27 van Bommel F, et al: Tenofovir for patients with lamivudine-resistant hepatitis $B$ virus (HBV) infection and high HBV DNA level during adefovir therapy. Hepatology 2006;44: 318-325.

28 Tenney DJ, et al: Clinical emergence of entecavir-resistant hepatitis B virus requires additional substitutions in virus already resistant to Lamivudine. Antimicrob Agents Chemother 2004;48:3498-3507.

29 Zoulim F, Locarnini S: Hepatitis B virus resistance to nucleos(t)ide analogues. Gastroenterology 2009;137:1593-1608.e1-2.

30 Zoulim F, Locarnini S: Management of treatment failure in chronic hepatitis B. J Hepatol 2012;56(suppl 1):S112-S122.

31 Seeger C, Mason WS: Hepatitis B virus biology. Microbiol Mol Biol Rev 2000;64:51-68.

32 Huang $\mathrm{H}$, et al: Structure of a covalently trapped catalytic complex of HIV-1 reverse transcriptase: implications for drug resistance. Science 1998;282:1669-1675.

33 Kohlstaedt LA, et al: Crystal structure at $3.5 \AA$ resolution of HIV-1 reverse transcriptase complexed with an inhibitor. Science 1992; 256:1783-1790.

34 Jacobo-Molina A, et al: Crystal structure of human immunodeficiency virus type 1 reverse transcriptase complexed with doublestranded DNA at 3.0 A resolution shows bent DNA. Proc Natl Acad Sci USA 1993;90:63206324.

35 Bartholomeusz A, Tehan BG, Chalmers DK: Comparisons of the HBV and HIV polymerase, and antiviral resistance mutations. Antivir Ther 2004;9:149-160.

36 Das K, et al: Molecular modeling and biochemical characterization reveal the mechanism of hepatitis B virus polymerase resistance to lamivudine (3TC) and emtricitabine (FTC). J Virol 2001;75:4771-4779.
Doublie S, Sawaya MR, Ellenberger T: An open and closed case for all polymerases. Structure 1999;7:R31-R35.

38 Steitz TA: DNA polymerases: structural diversity and common mechanisms. J Biol Chem 1999;274:17395-17398.

39 Tantillo C, et al: Locations of anti-AIDS drug binding sites and resistance mutations in the three-dimensional structure of HIV-1 reverse transcriptase. Implications for mechanisms of drug inhibition and resistance. J Mol Biol 1994;243:369-387.

40 Davis MG, et al: DNA polymerase activity of hepatitis B virus particles: differential inhibition by L-enantiomers of nucleotide analogs. Antiviral Res 1996;30:133-145.

41 Lee K, Chu CK: Molecular modeling approach to understanding the mode of action of L-nucleosides as antiviral agents. Antimicrob Agents Chemother 2001;45:138-144.

42 Yadav V, Chu CK: Molecular mechanisms of adefovir sensitivity and resistance in HBV polymerase mutants: a molecular dynamics study. Bioorg Med Chem Lett 2004;14:43134317.

43 Ballesteros JA, et al: Serine and threonine residues bend alpha-helices in the chi(1) $=\mathrm{g}(-)$ conformation. Biophys J 2000;79:2754-2760.

44 Nowak MA, et al: Viral dynamics in hepatitis $B$ virus infection. Proc Natl Acad Sci USA 1996;93:4398-4402.

45 Torresi J, et al: Reduced antigenicity of the hepatitis B virus HBsAg protein arising as a consequence of sequence changes in the overlapping polymerase gene that are selected by lamivudine therapy. Virology 2002;293:305313.

46 Kamili S, et al: Efficacy of hepatitis B vaccine against antiviral drug-resistant hepatitis $B$ virus mutants in the chimpanzee model. Hepatology 2009;49:1483-1491.

47 Clements CJ, et al: Global control of hepatitis $B$ virus: does treatment-induced antigenic change affect immunization? Bull World Health Organ 2010;88:66-73.

48 Warner N, Locarnini S: The antiviral drug selected hepatitis B virus rtA181T/sW172* mutant has a dominant negative secretion defect and alters the typical profile of viral rebound. Hepatology 2008;48:88-98.

49 Carman WF: The clinical significance of surface antigen variants of hepatitis B virus. J Viral Hepat 1997;4(suppl 1):11-20.

50 Liaw YF, et al: Asian-Pacific consensus statement on the management of chronic hepatitis: a 2012 update. Hepatol Int 2012;6:531561 